\title{
Idiopathic granulomatous mastitis: dilemmas in diagnosis and treatment
}

\author{
Rahim Mahmodlou ${ }^{1}$, Niloofar Dadkhah ${ }^{2}$, Fariba Abbasi ${ }^{3}$, Jafar Nasiri $^{4}$, Rohollah Valizadeh ${ }^{5}$
}

${ }^{1}$ M.D., Associate Professor in Thoracic and General Surgery, Department of Surgery, Imam Khomeini Hospital, Urmia University of Medical Sciences, Urmia, Iran

${ }^{2}$ Medical Student, Urmia University of Medical Sciences, Urmia, Iran

${ }^{3}$ M.D., Associate Professor in Pathology, Imam Khomeini Hospital, Urmia University of Medical Sciences, Urmia, Iran

${ }^{4}$ Doctor of Veterinary, Urmia University of Medical Sciences, Urmia, Iran

${ }^{5}$ M.Sc. Student of Epidemiology, Student Research Committee, Urmia University of Medical Sciences, Urmia, Iran

\section{Type of article: Original}

\begin{abstract}
Background: Idiopathic granulomatous mastitis (IGM) is a benign rare inflammatory disease of the breast. Due to its uncommon etiology, diagnosis and treatment is still unknown. Selection of a standard method for diagnosing idiopathic granulomatous mastitis is sophisticated. In view of non-definitive clinical and imaging finding, histopathology is the cornerstone of definitive diagnosis.

Objective: To determine and help solve the dilemma of treatment and diagnosis of idiopathic granulomatous mastitis.

Methods: This historical cohort study was conducted on 48 patients who referred to the general surgery clinic of Imam Khomeini Hospital of Urmia, were diagnosed with IGM and were histopathologically selected by census using the registry system, in Urmia city, Iran, during 2010-2015 so that medical reports, ultrasonography (US) and mammography (MMG) findings, follow-up information and recurrence rate were obtained from records. The data were analyzed using SPSS software version 18 and descriptive statistics were used.

Results: According to records, $68.75 \%$ of patients $(n=33)$ had palpable mass, $45.83 \%$ of patients $(n=22)$ had breast pain and swelling, erythema and $20.83 \%$ of patients $(n=10)$ had purulent drainage. Of the 48 patients 12 $(25 \%)$ had mammography, which revealed the following findings: mass with irregular border in 6 patients, skin thickness in 2 cases $(4.16 \%)$, and parenchymal asymmetry in 4 cases (8.33\%). Minimum follow-up was 24 (range 24-56) months.

Conclusions: According to our findings, histopathology of the disease is fundamental for correct diagnosis. Steroid therapy as a therapeutic method such as prednisolone was an effective and applicable choice in the treatment of idiopathic granulomatous mastitis by decreasing in inflammation.

Keywords: Breast, Granulomatous, Mastitis, Diagnosis, Therapy
\end{abstract}

\section{Introduction}

Idiopathic granulomatous mastitis is an uncommon, benign and chronic inflammatory breast disease that mainly involves women of childbearing age (1-4). The disease, was first described by Kessler and Wollock in 1972 (5). Although it is a benign condition, it is important from different points of view: 1 -it poses a diagnostic and treatment dilemma $(2,3)$, also, it clinically and mammographically mimics breast cancer, especially inflammatory type (4). On the other hand, due to lack of a definitive treatment plan, complications of empiric treatment, such as allergic reaction to antibiotics and poor cosmetic procedures result in following repeated surgical interventions which threaten the patients $(2,4)$. In fact, the etiology of IGM is unknown but some factors have been stated including reaction to chemical materials such as OCP, infectious disease, autoimmune diseases and immunologic response for

\section{Corresponding author:}

Rohollah Valizadeh, Student Research Committee, Urmia University of Medical Sciences, Urmia, Iran.

Tel: +98. 9142664745, Fax:+98.44334629, Email: rohvali4@gmail.com

Received: May 08, 2017, Accepted: August 05, 2017, Published: September 2017

iThenticate screening: June 29, 2017, English editing: August 27, 2017, Quality control: September 14, 2017

(C) 2017 The Authors. This is an open access article under the terms of the Creative Commons Attribution-NonCommercialNoDerivs License, which permits use and distribution in any medium, provided the original work is properly cited, the use is non-commercial and no modifications or adaptations are made. 
milk leakage from the breast's lobule $(3,6)$. On the other hand, some conditions such as pregnancy, breast feeding, hyperprolactinemia, galactorrhea, and alpha 1 antitrypsin deficiency have been associated with the risk of the disease $(4,6)$. Infection with corynebacterium kroppenstedtii has been suggested but is unconfirmed (6). The final word about etiology is that although the cause of the disease is unknown the general consensus is that reproductive age, recent pregnancy, breast feeding and history of OCP use are the most associated conditions with the disease, as shown in our finding. The most common presenting sign is a defined hard lump of the breast. As the disease progresses nipple inversion, peau d'orange, tumorous in duration, ulcer and fistula can occur that can easily be mistaken for cancer $(2,5,6)$. The most common reported ultrasound (U/S) findings are: 1 - an irregular hypo echoic finding that connects with the tubular hypo echo area and 2 - parenchymal heterogeneity and an area of mixed echo pattern with parenchymal deformity, both of which can lead to diagnosis of malignant changes $(4,6)$. Common findings in mammography imaging are asymmetric diffuse and skin thickness (4). As mentioned previously, neither U/S nor mammography can differentiate IGM from malignant or other benign lesions, especially inflammatory breast cancers $(4,5)$. Because of $\mathrm{U} / \mathrm{S}$ and mammographic failure, some authors suggested MRI in diagnosis of IGM, but studies have shown that MRI does not provide additional findings for differentiation of IGM from breast cancer (4). In view of the non-definitive clinical and imaging findings, histopathology is the cornerstone of definitive diagnosis $(5,6)$. It must be mentioned that FNA cannot confidently differentiate IGM and histopathological examination remains as the cornerstone $(5,6)$. Some studies obtained different results in terms of the treatment of these patients that suggested corticosteroids and methotrexate with surgery or treatment with corticosteroid and azithromycin, and administration of steroids in lesions in these patients (7-9). This study is aimed to help solve the dilemma of treatment and diagnosis of idiopathic granulomatous mastitis and answer to the question of which method can be useful for treating and diagnosing such patients, in fact we used the steroid therapy method for treating such patients and applied both MG and U/S for diagnosing such patients, and compared these methods together.

\section{Material and Methods}

\subsection{Research design and participants}

In this historical cohort study in Urmia city, Iran, from 2010 to 2015, 48 patients who referred to the general surgery clinic of Imam Khomeini Hospital in Urmia and were enrolled with idiopathic granulomatous mastitis accompanied with pathologic findings were selected by census using the registry system. Mean age of patients was 27 years. All of them had experienced at least one full term of pregnancy. Mean number of term of pregnancy was $1.65 \pm 0.128$. All patients had breast fed for a minimum of 6 months. All patients had used OCP with a minimum of 4 month before the first pregnancy. Furthermore, 46 patients had received antibiotics before pathologic confirmation. Four patients before our confirmation had undergone abscess drainage and abscess wall biopsy. In two of them granulomatous mastitis had been reported and an anti-tuberculosis (TB) drug was administrated and due to poor response of the drugs, they had referred to our clinic. In two cases inflammation and abscess formation was reported that in review of the specimen, IGM was confirmed in one case and in other case, re-biopsy was done and IGM was confirmed.

\subsection{Sign and symptoms}

According to records, $68.75 \%(\mathrm{n}=33)$ of patients had palpable mass, $45.83 \%(\mathrm{n}=22)$ had breast pain, swelling, erythema and $20.83 \%(n=10)$ had purulent drainage. Also, 2 patients were admitted to the rheumatology ward due to erythema nodosum (Figure 1) and surgery consultation was done due to their breast mass. On review of their history and medical records, it was revealed that both of them, had gone to a clinic due to breast symptoms as outpatients and cloxacillin or cephalosporin had been prescribed for both. In both of them, biopsy confirmed IGM. In one of them, who was pregnant in first trimester, we could not give a high dose of steroid and prednisolone $10 \mathrm{mg}$ daily started according to gynecology and rheumatology consultation, unfortunately she did not responded to low dose steroid and multiple abscess occurred (Figure 2). The second case responded to high dose prednisolone ideally.

\subsection{Treatment}

The importance of completing the course of treatment was explained to all patients. Patients were told that the cornerstone of treatment is steroids. Complications of steroid therapy such as obesity, glucose intolerance, peptic ulcer disease and acne was explained to them and their husbands. Failure of low dose steroids was reported in previous studies, in one of our patients (who was pregnant), for this reason we began treatment with high dose steroids. Dosage of prednisolone gradually decreased from prednisolone $50 \mathrm{mg}$ BID for two weeks, $50 \mathrm{mg}$ daily for two weeks, $25 \mathrm{mg}$ daily for 4 weeks, $10 \mathrm{mg}$ daily for 2 weeks and $5 \mathrm{mg}$ for one week, respectively. Cap cloxacillin 
$500 \mathrm{mg}$ QID and cap clindamycin $500 \mathrm{mg}$ were prescribed besides prednisolone for two weeks also, tab ranitidine $150 \mathrm{mg}$ daily was prescribed in conjunction with $300 \mathrm{mg}$ TDS steroid consumption.

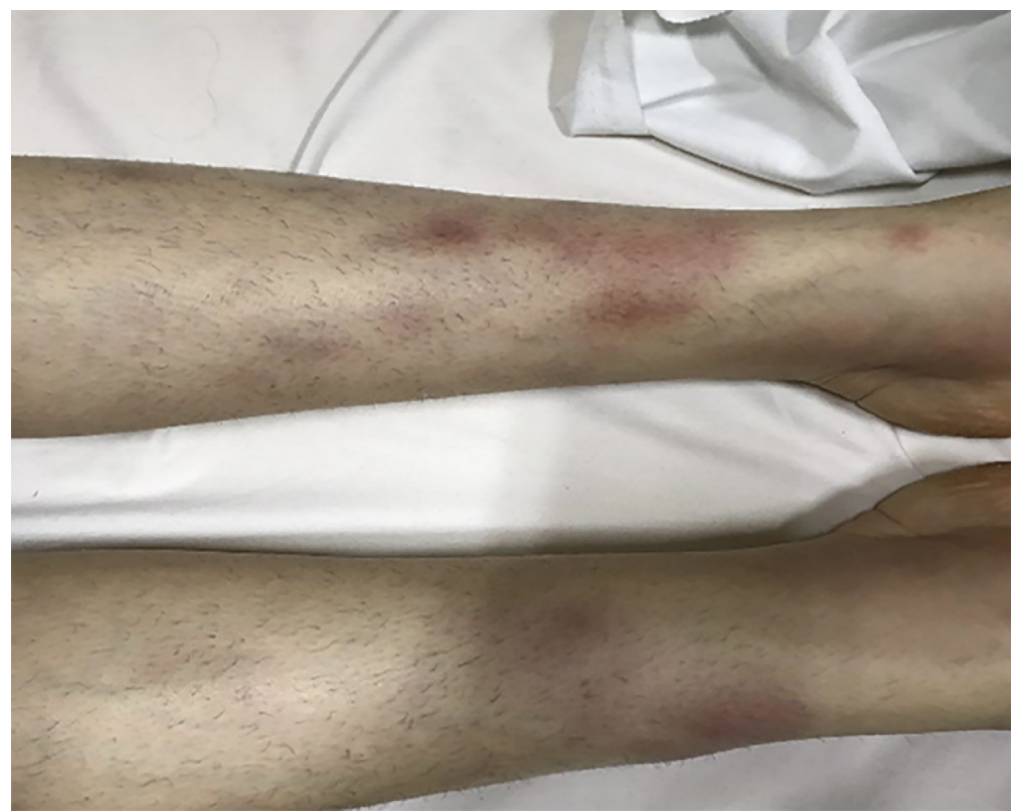

Figure1. Erythema nodusum of patient after use of cephalosporin.

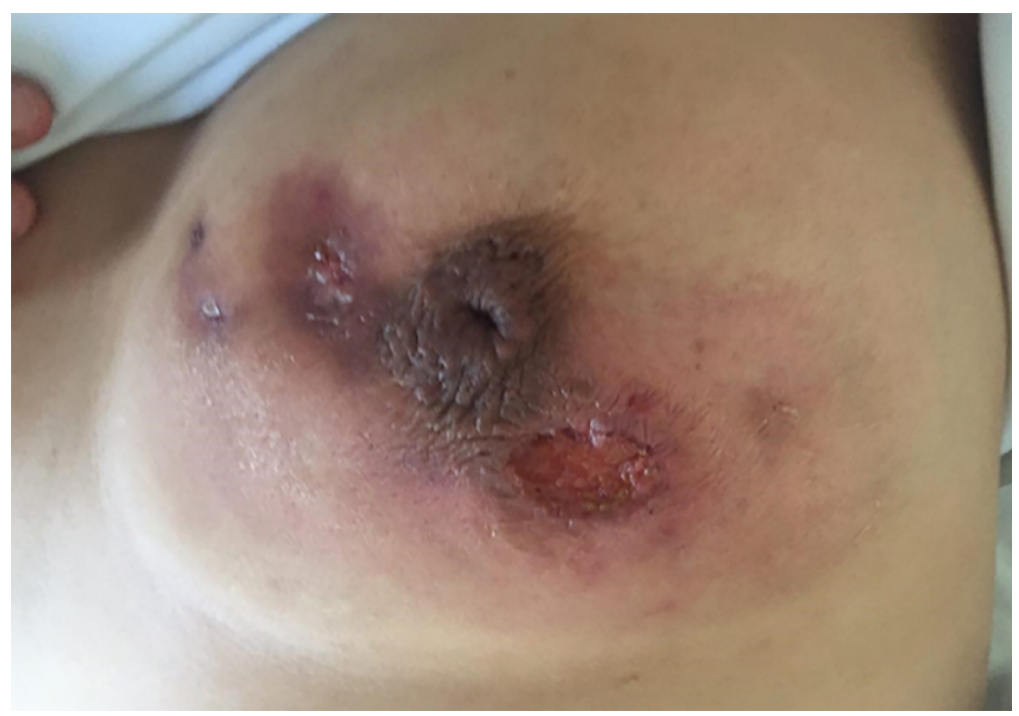

Figure2. Abscess of breast despite use of low dose prednisolone.

\subsection{Follow up}

All patients were visited every two weeks and were recommended to refer to the hospital as soon as they experienced a complication. After consultation with the prenatologist, one patient who was pregnant required low doses of prednisolone $10 \mathrm{mg}$ daily. Unfortunately, her response was not good and she had multiple abscesses in late pregnancy (Figure 2). Moreover, 2 of 47 patients discontinued the treatment due to obesity, and acne without coming back to hospital 4 to 6 weeks after the beginning of treatment. Both of them returned 3 to 4 weeks later with draining abscess and we had to remove the necrotic skin. One of the patients, one week after treatment, came back with erythema nodosum and arthralgia signs and the patient had to be hospitalized. The mentioned patients were treated with erythromycin (discontinuation of cloxacillin and replacement with erythromycin) without discontinuation of other drugs that caused no problem in follow-up. This study was approved by the Ethics Committee of Urmia University of Medical Sciences and the goals of the study were explained to all participants of 
whom all agreed to participate, and were assured regarding the confidentiality of their individual information as well as the voluntary nature of participating in the study. The data were analyzed using SPSS software version 18 (SPSS Inc., Chicago, Illinois, USA) and descriptive statistics were used.

\section{Results}

All patients (excluding one pregnant patient) were followed up for at least 24 months (24-56 months), they were visited every 14 days until they were under treatment protocol, after that, every 3 months until 12 months and finally every 6 months. During this period of follow up, only 3 patients experienced recurrence, including pain and firmness in the biopsy site in one patient and erythema with collection in the same breast but other quadrant in two patients. IGM was reconfirmed in, the first patient with a core needle biopsy, and in the two other patients, drainage and open biopsy respectively. These three patients were treated with another course and followed up for 36 months as with others, without recurrence. A total of 12 out of 48 patients $(25 \%)$ had mammography, with the following findings: mass with irregular border in 6 patients, skin thickness in 2 cases $(4.16 \%)$, and parenchymal asymmetry in 4 cases $(8.33 \%)$. Ultrasound had been done in 38 patients $(79.16 \%)$ with the following findings: Hypo echo mass in 18 patients $(16.66 \%)$, irregular mass in 6 patients $(12.5 \%)$. Echogenic collection in 6 patients $(12.5 \%)$, inflammatory changes in 3 patients $(6.25 \%)$, abscess in 3 patients $(6.25 \%)$ and parenchymal heterogeneity in 2 patients $(4.16 \%)$.

\section{Discussion}

Idiopathic granulomatous mastitis is an uncommon, benign and chronic inflammatory breast disease that mainly involves women in childbearing age (1-4). The disease was first described by Kessler and Wollock in 1972 (5). Although it is a benign condition it is important from different points of view: 1-it poses a diagnostic and treatment dilemma $(2,3)$ and 2 - it clinically and mammographically mimics breast cancer especially inflammatory type (4). Tissues for histopathological examination can be obtained with core needle biopsy, excisional biopsy and incisional biopsy of abscess wall in patients requiring drainage (4-6). Characteristic pathologic findings include chronic granulomatous lobulitis with caseous necrosis plus giant cell, leukocytosis, epithelioid cells, macrophages and micro abscess $(2,4,6)$. Although absence of caseous necrosis dose not exclude the diagnosis of T.B, it must be mentioned that T.B mastitis is a rare disease, aside from this, isolated involvement of the breast is very rare (5). Nevertheless, we believe that tissue culture and staining is necessary to exclude T.B. infection especially in endemic regions, as we had done in the present study (5). In the study of Atak et al., surgical excision with administration of steroids in lesions were the best treatment method for IGM patients. In this study, 2 patients $(5 \%)$ encountered with erythema nodosum, 6 patients $(15 \%)$ had nipple retraction and 9 patients $(22.5 \%)$ had abscess as well as the most common complaint which was breast mass (55\%) (9). Complexity in treatment is more than in diagnosis because in contrast to diagnosis, there is no definitive treatment (2, 4, and 5). Available options include observation, antibiotics, steroids, immunosuppressive and surgical treatment $(5,6)$. In the study of Salehi et al., in 2013granulomatous mastitis was investigated in Alzahra hospital. The mean age of patients was $33.6 \pm 8.9$. Of a total of 26 patients, 24 patients $(92.3 \%)$ were treated with corticosteroid and azithromycin for IGM (8). These results were consistent with our results. As well as in the study of Sheybani et al., conducted on 22 patients with idiopathic granulomatous mastitis, corticosteroids plus methotrexate, with surgery were the treatment of choice in these patients (7). Although antibiotics are widely used, there is no proven benefit in studies (5), as our study showed that only 3 of 21 were resolved with antibiotics alone. It is suggested that uncomplicated cases of IGM can be observed without treatment (2-6). In our study, we did not use this option. Although, in most cases that present with erythema and other signs that mimic infection, usually systemic antibiotics are started empirically (2), as we conducted in the present study. Nevertheless, there is proven benefit in most studies improvement with less than 5\% (5). Most studies suggest that after ruling out the infectious processes, oral steroids are necessary $(2,5,6)$. The steroid sparing agents such as methotrexate or azathioprine may be used for several reasons; to facilitate tapering of steroids, in cases resistance to steroids, or recurrence after discounting steroids $(2-4,6)$. We recommended steroid therapy in high doses to our patients after abscess drainage and antibiotics. Various studies had been conducted regarding the effect of steroids that confirmed its effect over a long period of time. In the study of Sakurai et al., complete resolution of mass lesions with corticosteroid was observed from 4 to 10 months (10). There is no accepted optimal and standard treatment for IGM and more randomized trials and cohort studies related to method of treatment and diagnosis to solve this challenge of medical sciences are required.

\section{Limitations and key points of the study}

The small sample size of included studies are potential limitation of this study. Other limitations of the current study, were small sample size of this study. There is still need for further studies to access additional information about this subject. Key points of this study were 1) the prevalence of idiopathic granulomatous mastitis has 
increased recently, especially in developing countries, 2) a high dose of steroid therapy is the choice treatment for IGM, if possible, 3) both mammography and ultrasound are useful for diagnosis of idiopathic granulomatous mastitis, 4) abscess of breast's lobule was aroused after use of prednisolone in patient, 5) erythema of patient was aroused after use of prednisolone in patient.

\section{Conclusions}

According to our findings, histopathology of the disease is fundamental for correct diagnosis. Steroid therapy, as a therapeutic method such as prednisolone, was an effective and applicable choice in the treatment of idiopathic granulomatous mastitis by decreasing in inflammation. There is no acceptable method of treatment for IGM. We found that high-dose steroid therapy is the choice treatment for IGM. Its prevalence has increased recently especially in developing countries, and it seems that comprehensive studies are necessary to specify etiology and to determine the best treatment of this disease.

\section{Acknowledgments:}

This research was extracted from a doctorate thesis (grant: 1394-01-32-2117) and was financially supported by the Research Council of Urmia University of Medical Sciences and the authors appreciate that unit.

\section{Conflict of Interest:}

There is no conflict of interest to be declared.

Authors' contributions:

All authors contributed to this project and article equally. All authors read and approved the final manuscript.

\section{References:}

1) Bellavia M, Damiano G, Palumbo VD, Spinelli G, Tomasello G, Marrazzo A, et al. Granulomatous Mastitis during Chronic Antidepressant Therapy: Is It Possible a Conservative Therapeutic Approach? J Breast Cancer. 2012; 15(3): 371-2. doi: 10.4048/jbc.2012.15.3.371. PMID: 23091553, PMCID: PMC3468794.

2) Garcia-Rodiguez JA, Pattullo A. Idiopathic granulomatous mastitis: a mimicking disease in a pregnant woman: a case report. BMC Res Notes. 2013; 6: 95. doi: 10.1186/1756-0500-6-95. PMID: 23497626, PMCID: PMC3606122.

3) Kalaycı TÖ, Koruyucu MB, Apaydın M, Etit D, Varer M. Idiopathic Granulomatous Mastitis Associated with Erythema Nodosum. Balkan Med J. 2016; 33(2): 228-31. PMID: 27403395, PMCID: PMC4924970.

4) Gurleyik G, Aktekin A, Aker F, Karagulle H, Saglamc A. Medical and Surgical Treatment of Idiopathic Granulomatous Lobular Mastitis: A Benign Inflammatory Disease Mimicking Invasive Carcinoma. J Breast Cancer. 2012; 15(1): 119-23. doi: 10.4048/jbc.2012.15.1.119. PMID: 22493638, PMCID: PMC3318163.

5) Ergin $\mathrm{AB}$, Cristofanilli $\mathrm{M}$, Daw $\mathrm{H}$, Tahan $\mathrm{G}$, Gong Y. Recurrent granulomatous mastitis mimicking inflammatory breast cancer. BMJ Case Rep. 2011; 2011. doi: 10.1136/bcr.07.2010.3156. PMID: 22715267, PMCID: PMC3062046.

6) Patel RA, Strickland P, Sankara IR, Pinkston G, Many W, Rodriguez M. Idiopathic Granulomatous Mastitis: Case Reports and Review of Literature. J Gen Intern Med. 2010; 25(3): 270-3. doi: 10.1007/s11606-009-1207-2. PMID: 20013067, PMCID: PMC2839326.

7) Sheybani F, Sarvghad M, Naderi HR, Gharib M. Treatment for and clinical characteristics of granulomatous mastitis. Obstet Gynecol. 2015; 125(4): 801-7. doi: 10.1097/AOG.0000000000000734. PMID: 25751209.

8) Salehi M, Salehi M, Kalbasi N, Hakamifard A, Salehi H, Salehi MM, et al. Corticosteroid and Azithromycin in Idiopathic Granulomatous Mastitis. Adv Biomed Res. 2017; 6: 8. PMID: 28217653, PMCID: PMC5309447.

9) Atak T, Sagiroglu J, Eren T, Ali Ozemir I, Alimoglu O. Strategies to treat idiopathic granulomatous mastitis: retrospective analysis of 40 patients. Breast Dis. 2015; 35(1): 19-24. PMID: 24989362.

10) Sakurai K, Fujisaki S, Enomoto K, Amano S, Sugitani M. Evaluation of follow-up strategies for corticosteroid therapy of idiopathic granulomatous mastitis. Surg Today. 2011; 41(3): 333-7. doi: 10.1007/s00595-009-4292-2. PMID: 21365412. 